\title{
Laparoscopic Hernioplasty Using Omega-3 Coating Mesh
}

\author{
Masahiko Kawaguchi* , Norihiko Ishikawa, Youji Nishida, Hideki Moriyama, \\ Masahiro Kaneki, Go Watanabe \\ Department of General and Cardiothoracic Surgery, Kanazawa University, Kanazawa, Japan \\ Email: "surgkw@gmail.com
}

Received June 17, 2012; revised July 20, 2012; accepted August 5, 2012

\begin{abstract}
Background: Laparoscopic hernioplasty has gained popularity with significant advances in prostheses. Omega-3 coating mesh (C-Qur) is a prosthesis that can be used in the abdominal cavity, and the coating prevents adhesion of the mesh to the viscera. We planned a prospective observational study of laparoscopic hernioplasty using C-Qur. Methods: C-Qur was used in laparoscopic hernioplasty over the course of 1 year. We considered laparoscopic approaches as our primary treatment method for abdominal wall hernias. Although only a single incision was made for the majority of the laparoscopic hernioplasties, additional incisions were made when severe adhesions were encountered. For incisional or ventral hernias, a lateral lower incision was made. For inguinal hernias, an umbilical incision was made. Sex, age, diagnosis, number of incisions, additional incisions, morbidity, and follow-up period were evaluated. Results: Twenty-four patients who underwent surgery between May 2010 and April 2011 were included in this study. The median follow-up period was 14 months. The most common early complications included wound pain and edema; however, there were no persistent complications.
\end{abstract}

Keywords: Omega-3 Coating Mesh; Single Incision Laparoscopic Surgery; Laparoscopic Hernioplasty; Inguinal Hernia; Incisional Hernia

\section{Introduction}

The use of laparoscopic hernioplasty is spreading [1,2], and the use of prostheses is also gaining popularity in line with the advancement of prostheses and laparoscopic devices. Omega-3 coating mesh (C-Qur; Atrium Medical Corp, Hudson, NH, USA) is one of these newly developed prostheses, and it has unique properties because of its absorbable barrier coating. The mesh can be placed directly on the peritoneum in the abdominal cavity, and the omega- 3 coating prevents adhesion of the mesh to the viscera, thus promoting reperitonealization on the mesh.

We have been performing hernioplasty using C-Qur since May 2010; however, clinical evaluation of hernioplasty performed using C-Qur has not yet been published. Therefore, we studied our experiences with laparoscopic hernioplasty performed using C-Qur and present the results herein.

\section{Patients and Methods}

\subsection{Patients Characteristic}

Laparoscopic hernioplasties using C-Qur were performed from May 2010 to April 2011 at the department of General and Cardiothoracic Surgery of Kanazawa University,

${ }^{*}$ Corresponding author. and were assessed by prospective observational study.

Laparoscopic surgery was indicated for all incisional, ventral and inguinal hernia patients who did not have pre-existing health conditions that would prevent safe anesthesia or could not discontinue anticoagulant agents, whether or not a history of abdominal surgeries existed. Most of them involved an intended single incision method; however, when a severe adhesion was encountered, additional incisions were made. Young women of a reproductive age were excluded from the study, because of possibility of adverse effects for pregnancy.

\subsection{Surgical Technique}

For incisional hernias, a lateral lower incision was made, avoiding the location of any previous incisional scars. Following laparoscopy, hernia contents were reduced and adhesiolysis was performed to clear the hernia orifice. C-Qur was then cut to a size large enough to cover a hernia orifice by $3 \mathrm{~cm}$ or more around the rim. The C-Qur was then anchored at 4 points with trans-fascial sutures by using 3-0 Vicryl. Finally, C-Qur was fixed to the peritoneum by using absorbable tacks around the rim (Figure 1).

For inguinal hernias, an umbilical incision was made. After confirming the diagnosis, the sac was retracted and 
peeled away from the surrounding tissues, including the spermatic chord and testicular blood vessels. A continuous incision was made till the bottom of the hernia sac. The orifice of the hernia and Hesselbach's triangle were measured in order to prepare the C-Qur. If necessary, an incision was made to the ipsilateral medial umbilical fold in order to attach the C-Qur to the hernia. Fixation of the C-Qur to the peritoneum was achieved using absorbable tacks (Figure 2).

\subsection{Assessment and Postoperative Care}

Data were accumulated for age, sex, diagnosis, comorbidity, complications during the procedure, early postoperative morbidity, late morbidity, and follow-up period. All patients received loxoprofen sodium for 3 days after the operation, and additional analgesic agents were administered for postoperative pain.

\subsection{Statistical Analysis}

Statistical analysis was performed using the R 2.12 software. Numerical data were given as median with the range and categorical variables were expressed by number (n).

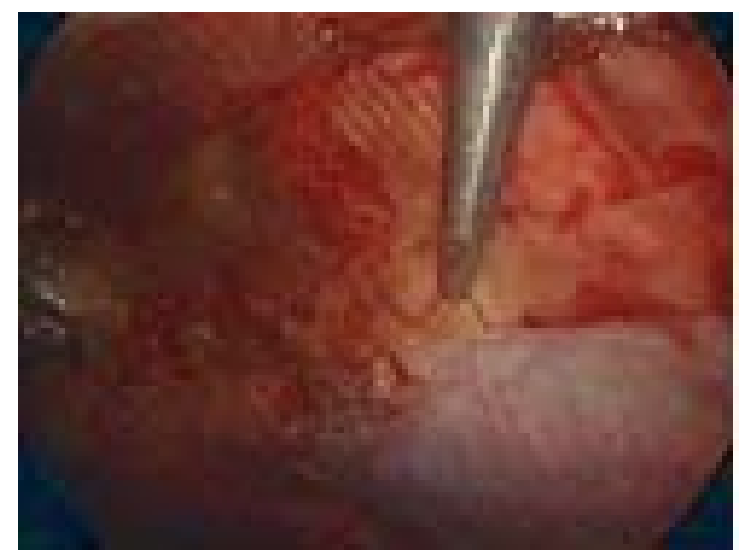

Figure 1. Incisional hernioplasty using omega-3 coating mesh.

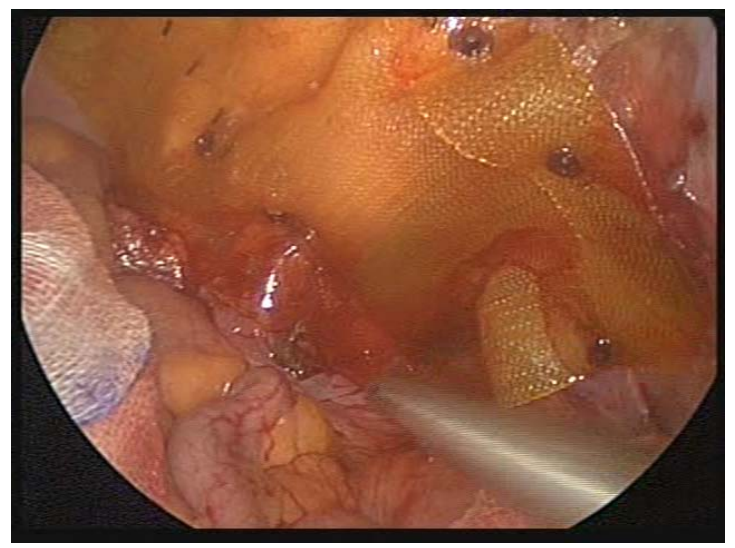

Figure 2. Right inguinal hernioplasty using omega-3 coating mesh.

\section{Results}

Hernioplasty using C-Qur was performed in 26 cases during the study period. Here, we describe 24 cases in which laparoscopic hernioplasty was performed (Table 1).

The median age was 69 years (range, from 25 to 85 years), and the numbers of male and female patients were 22 and 2, respectively. Of these, those female patients had incisional hernia and obturator hernia. In total, there were 4 cases of incisional or ventral hernias, 19 cases of inguinal hernias, and 1 case of obturator hernia. There were no incidences of severe bleeding or any visceral injury during the procedures. There were no cases that needed to be converted to open surgery.

Incisional and ventral hernia cases are summarized in Table 2. In 2 cases, an additional incision was needed while performing adhesiolysis. Inguinal hernia cases are summarized in Table 3 . Additional incisions were made in 3 cases, and in 1 case, the positions of the 3 ports were

Table 1. Demographic characteristics of patients who underwent laparoscopic hernioplasty using omega-3 coating mesh.

\begin{tabular}{cc}
\hline Age & $\begin{array}{c}\text { Median age 69 years } \\
\text { (range, 25 - 85 years) }\end{array}$ \\
\hline Sex & $22: 2$ \\
Diagnosis & 4 cases \\
$\begin{array}{c}\text { Incisional or ventral hernia } \\
\text { Inguinal hernia }\end{array}$ & 19 cases \\
Obturator hernia & 1 case \\
Previous laparotomy & 6 cases \\
Intended number of incisions & 19 cases $^{\mathrm{a}}$ \\
Single & 1 case \\
Two & 3 cases \\
Three & 1 cases \\
Four & 0 cases \\
Intraoperative complication & 5 cases \\
Early postoperative morbidity & 8 cases \\
Pain & 1 case \\
Swelling & 0 case \\
Repeat laparotomy &
\end{tabular}

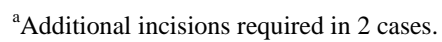

Table 2. Characteristics of cases with incisional and ventral hernias.

\begin{tabular}{ccccc}
\hline Sex & Age & Diagnosis & Ports & $\begin{array}{c}\text { Comorbidity } \\
\text { (Early morbidity) }\end{array}$ \\
\hline F & 54 & Umbilical hernia & 2 & $\begin{array}{c}\text { BMI 37.8 } \\
\text { (Skin ulcer, edema) }\end{array}$ \\
M & 62 & Incisional hernia & 1 & CRF \\
M & 82 & Incisional hernia & 2 & Aortic dissection \\
& & & & (Edema) \\
M & 71 & Incisional hernia & 1 & Asthma \\
\hline
\end{tabular}

F: female; M: male; BMI: body mass index; CRF: chronic renal failure. 
the same as those in the patients' previous laparoscopic procedures. Among the inguinal hernia cases, adhesion of the sigmoid to the left inguinal lesion was found in 3 cases. The obturator hernia was an emergency case without necrosis, and 4 ports were made.

Early postoperative complications included pain and edema (Table 1). In 1 case of ventral hernia, skin necrosis was observed at the umbilicus and dermatological therapy was required. The necrosis resolved after 1 month, without infection.

Repeat laparoscopy was performed because of suspected recurrence in 1 case of large inguinal hernia. However, no recurrence was found. The median follow-up period was 7 months (range, from 1 to 12 months), and no subsequent operations were performed. There was no persistent pain, persistent edema, bowel obstruction, infections, or any recurrent hernias.

\section{Discussions}

Laparoscopic hernioplasty using C-Qur is a safe, useful, and easy procedure. In our study, some early complications were observed, however, severe complications were not found. Since the prosthesis can be placed on the inner side of peritoneum, it provides an easy way to repair any hernia.

C-Qur is a polypropylene surgical mesh coated with omega-3 fatty acid, the catalytic properties of which prevent adhesion and reperitonealization on the mesh. Experiments showed that the use of C-Qur resulted in a minimal amount of contraction and a favorable tissue response 120 days after intraperitoneal placement [3]. Seven days after implantation, adhesion was not observed; however, adhesion was observed 30 days following implantation in another report [4]. We did not observe any complications related to the adhesion between the viscera and the mesh.

Table 3. Demographic characteristics of patients who underwent laparoscopic hernioplasty using omega- 3 coating mesh.

\begin{tabular}{cc}
\hline Sex & $19: 0$ \\
\hline Operation side & \\
Right & 4 cases \\
Bilateral & 11 cases \\
Left & 4 cases \\
Ports & \\
Single & 15 cases \\
Two & 1 case \\
Tree & 1 case \\
Previous laparotomy & 3 cases \\
After prostate cancer surgery & 2 cases \\
After other surgery & 5 cases \\
Postoperative edema & 0 case \\
Hematoma & 11 cases \\
Postoperative pain within self & \\
torelance & Median 2.5 days (range, 1 - 11 days) \\
Painduration
\end{tabular}

For the treatment of incisional hernia, a laparoscopic procedure using prosthesis is an easy and steady technique, and therefore, the procedure is increasingly becoming popular [5]. C-Qur is one of the prostheses used for the purpose. C-Qur has unique reperitonealization abilities, and thus, its use is more feasible for long-term prognosis. The other laparoscopic procedure for incisional hernia is component separation [6]. Although the procedure can be performed without prostheses, it is more complicated and the strength of the repaired abdominal wall relies solely on the endogenous tissues.

Thus, in the absence of an elevated risk of infection, hernioplasty using C-Qur is recommended for incisional hernia.

For inguinal hernia, hernioplasty using C-Qur is easier and simpler, allowing for a single incision laparoscopic surgery. However, the transabdominal preperitoneal (TAPP) or total extraperitoneal (TEP) approaches are more popular for laparoscopic hernioplasty $[7,8]$. In both approaches, a prosthesis is placed in the preperitoneal space because direct attachment between the viscera and a prosthesis may cause severe complications. However, C-Qur promotes reperitonealization and protects the viscera from direct attachment to the mesh. Additionally, although TAPP or TEP is needed more skilled technique [2], they may cause persistent inguinal pain or discomfort $[9,10]$. On the other hand, there was no persistent pain in our series.

\section{Conclusion}

Laparoscopic hernioplasty using C-Qur for incisional and inguinal hernias is a safe and easy procedure. Hernioplasty using C-Qur is a minimally invasive procedure that promotes a more natural recovery of the patients.

\section{REFERENCES}

[1] K. Breuing, C. E. Butler, S. Ferzoco, M. Franz, C. S. Hultman, J. F. Kilbridge, M. Rosen, R. P. Silverman and D. Vargo, "Incisional Ventral Hernias: Review of the Literature and Recommendations Regarding the Grading and Technique of Repair,” Surgery, Vol. 148, No. 3, 2010, pp. 544-558. doi:10.1016/j.surg.2010.01.008

[2] J. Carter and Q.-Y. Duh, "Laparoscopic Repair of Inguinal Hernias,” World Journal of Surgery, Vol. 35, No. 7, 2011, pp. 1519-1525. doi:10.1007/s00268-011-1030-x

[3] R. A. Pierce, J. M. Perrone, A. Nimeri, J. A. Sexton, J. Walcutt, M. M. Frisella and B. D. Matthews, "120-Day Comparative Analysis of Adhesion Grade and Quantity, Mesh Contraction, and Tissue Response to a Novel Omega-3 Fatty Acid Bioabsorbable Barrier Macroporous Mesh after Intraperitoneal Placement,” Surgical Innovation, Vol. 16, No. 1, 2009, pp. 46-54. doi:10.1177/1553350608330479

[4] M. H. Schreinemacher, P. J. Emans, M. J. Gijbels, J. M. Greve, G. L. Beets and N. D. Bouvy, "Degradation of 
Mesh Coatings and Intraperitoneal Adhesion Formation in an Experimental Model," British Journal of Surgery, Vol. 96, No. 3, 2009, pp. 305-313. doi:10.1002/bjs.6446

[5] S. Sauerland, M. Walgenbach, B. Habermalz, C. M. Seiler and M. Miserez, "Laparoscopic versus Open Surgical Techniques for Ventral or Incisional Hernia Repair," Cochrane Database Systematic Reviews, Vol. 16, No. 3, 2011, Article ID: CD007781.

[6] O. M. Ramirez, E. Ruas and A. L. Dellon, “'Components Separation' Method for Closure of Abdominal-Wall Defects: An Anatomic and Clinical Study," Plastic and Reconstructive Surgery, Vol. 86, No. 3, 1990, pp. 519-526. doi:10.1097/00006534-199009000-00023

[7] S. A. Kapiris, W. A. Brough, C. M. Royston, C. O’Boyle and P. C. Sedman, "Laparoscopic Transabdominal Preperitoneal (TAPP) Hernia Repair. A 7-Year Two-Center Experience in 3017 Patients," Surgical Endoscopy, Vol. 15,
No. 9, 2001, pp. 972-975. doi:10.1007/s004640080090

[8] E. Kuhry, R. N. van Veen, H. R. Langeveld, E. W. Steyerberg, J. Jeekel and H. J. Bonjer, “Open or Endoscopic Total Extraperitoneal Inguinal Hernia Repair? A Systematic Review,” Surgical Endoscopy, Vol. 21, No. 2, 2007, pp. 161-166. doi:10.1007/s00464-006-0167-4

[9] G. Linderoth, H. Kehlet, E. K. Aasvang and M. U. Werner, "Neurophysiological Characterization of Persistent Pain after Laparoscopic Inguinal Hernia Repair,” Hernia, Vol. 15, No. 5, 2011, pp. 521-529. doi:10.1007/s10029-011-0815-z

[10] R. Bittner, B. J. Leibl, B. Kraft and J. Schwarz, "OneYear Results of a Prospective, Randomised Clinical Trial Comparing Four Meshes in Laparoscopic Inguinal Hernia Repair (TAPP),” Hernia, Vol. 15, No. 5, 2011, pp. 503510. doi:10.1007/s10029-011-0810-4 